\title{
Parâmetros Reprodutivo e Metabólico de Vacas da Raça Guzerá Suplementadas no Pré e Pós-Parto ${ }^{1}$
}

\author{
Marcelo Marcondes de Godoy, 2,6 , João Batista Alves ${ }^{3}$, Alda Lúcia Gomes Monteiro4, Walter \\ Veriano Valério Filho ${ }^{5}$
}

\begin{abstract}
RESUMO - Foram utilizadas 30 vacas da raça Guzerá (10 por tratamento), suplementadas no pré-parto (PRE), pós-parto (POS) e sem suplementação (SS) com os objetivos de avaliar no pós-parto as variações do peso corporal (PC), do escore de condição corporal (ECC) $(1$ = muito magra a 9 = muito gorda) e dos níveis plasmáticos de colesterol total (NPCT), o índice de prenhez (IP) até 112 dias pós-parto e a viabilidade econômica dos tratamentos PRE e POS, que constou de $1 \mathrm{~kg} / \mathrm{dia} / \mathrm{animal}$ de concentrado (16\% PB; $3000 \mathrm{kcal}$ ED/kg). O PC e o ECC foram obtidos a cada 28 dias, e colheitas de sangue semanalmente para análise dos NPCT até 112 dias pósparto. O PC e o ECC tiveram interação dias pós-parto x tratamento. O tratamento PRE aumentou o PC e o ECC das vacas ao parto: $477,80 \mathrm{~kg}$ e 6,20 respectivamente, em comparação com os tratamentos POS e SS de 453,37 kg e 5,5; 447,57 kg e 5,28, respectivamente. O PC e o ECC do tratamento PRE foi superior de 28 a 84 dias comparado ao SS, enquanto o tratamento POS foi superior apenas de 84 a 112 dias pós-parto. O tratamento POS não diferiu do PRE no PC de 56 a 112 dias pós-parto e no ECC de 28 a 112 dias pósparto. O tratamento POS proporcionou aumento do PC e ECC das vacas, já os tratamentos PRE e SS perderam PC e ECC até 112 dias pós-parto. O PC e ECC dos tratamentos PRE, POS e SS aos 112 dias pós-parto foram de 457,79 kg e 5,38; 461,87 kg e 5,66; $436,57 \mathrm{~kg}$ e 4,78, respectivamente. Os NPCT tiveram efeito dos dias pós-parto, mas não de tratamento, aumentando com os dias pósparto. Os IP não foram influenciados pelos tratamentos PRE, POS e SS com 50,00; 62,20 e 57,14\%, respectivamente. Economicamente a suplementação não foi viável.
\end{abstract}

Palavras-chave: bovino, colesterol total, escore de condição corporal, nutrição, reprodução, peso corporal

\section{Reproductive and Metabolic Parameters of Guzerá Cows Supplemented in Pre and Postpartum}

\begin{abstract}
Thirty Guzera cows (10 by treatment), supplemented in the prepartum (PRE) and postpartum (POS) and without supplementation (SS) were used to evaluate, in the postpartum, the variation of body weight (BW), body condition score (BCS) $(1=$ very thin to $9=$ very fat) and levels of serum total cholesterol (TC), rate pregnancy at 112 days postpartum and economic viability of PRE and POS treatments, where the animals fed $1 \mathrm{~kg} /$ day/animal of concentration $(16 \% \mathrm{CP} ; 3000 \mathrm{kcal} \mathrm{DE} / \mathrm{kg})$. BW and the ECC were evaluated every 28 days, and blood collections were weekly performed for TC analysis until 112 days postpartum. Days postpartum x treatment interaction was observed for BW and ECC. PRE treatment increased the BW and ECC of the cows at parturition: $477.80 \mathrm{~kg}$ and 6.20, respectively, in relation to POS and SS treatments, of the $453.37 \mathrm{~kg}$ and 5.5, 447.57 kg and 5.28, respectively. treatment PRE showed highr values of BW and ECC from 28 to 84 days, when compared to SS, while POS treatment shwed higher values from 84 to 112 days postpartum. POS treatment did not differ from PRE in BW from 56 to 112 days postpartum and in ECC from 28 to 112 days postpartum. BW and ECC increased in the POS treatment and BW and ECC decreased in the PRE and SS treatments until 112 dayspostpartum. BW and ECC in the PRE, POS and SS treatments at 112 days postpartum were $457.79 \mathrm{~kg}$ and 5.38, $461.87 \mathrm{~kg}$ and 5.66, 436.57 kg and 4.78, respectively. TC showed days postpartum effect, but not of treatment, and increased as the postpartum days increased. RP was not influenced by PRE, POS and SS treatments $(50.00,62.20$, and $57.14 \%$, respectively). The supplementation was not viable.
\end{abstract}

Key Word: body condition score, cattle, body weight, nutrition, reproduction, total cholesterol

\footnotetext{
${ }^{1}$ Parte da dissertação do primeiro autor apresentada à FEIS/UNESP - Ilha Solteira.

2 Médico Veterinário, Mestre em Zootecnia. E.mail: m.m.godoy@bol.com.br

3 Prof. Departamento de Biologia e Zootecnia, FEIS/UNESP - Ilha Solteira, Av. Brasil Centro, 56 - Cx. Postal 31 - CEP: $15385-000$. E.mail: jbalves@bio.feis.unesp.br

4 Universidade Federal do Paraná, Setor de Ciências Agrárias, Rua dos Funcionários, 1540, Bairro: Cabral, Curitiba-PR, CEP: 80035-050 ${ }^{5}$ Prof. Departamento de Matemática, FEIS/UNESP-Ilha Solteira.

${ }^{6}$ Bolsista CAPES.
} 


\section{Introdução}

A intensificação dos sistemas de produção dos bovinos de corte, com o intuito de aumentar os índices produtivos, demanda por tecnologias que favoreçam a exploração racional da pecuária de corte. A eficiência reprodutiva, reflexo das condições de nutrição, manejo e sanidade, juntamente com a qualidade genética, são consideradas características importantes, influenciando no desfrute geral do rebanho. Entre os inúmeros fatores que afetam o desempenho reprodutivo de bovinos, a nutrição é talvez aquele que tem maior impacto (Santos \& Amstalden, 1998). Rebanhos com nutrição inadequada apresentam baixos índices reprodutivos atrasando o reinicio da atividade ovariana luteal cíclica no pós-parto, o que poderia ser evitado ou amenizado pela suplementação nutricional durante o inverno (Stabenfeldt, 1993).

O intervalo da parição ao primeiro cio pós-parto é denominado anestro (Pires \& Susin, 1997), cuja taxa é influenciada pelo manejo nutricional e a amamentação, além de outros fatores como estação do ano, raça, idade e presença do touro (Short et al., 1990; Oliveira Filho, 2002). A amamentação controlada pode aumentar os índices de prenhez ao bloquear os efeitos deletérios da amamentação sobre a atividade ovariana, através de neuro hormônios liberados via sucção dos tetos pelos bezerros (Freitas, 2000).

$\mathrm{O}$ anestro nutricional ocorre quando há restrição alimentar ou consumo insuficiente de nutrientes para atender às exigências do animal. A energia tem sido considerada o fator predisponente determinante mais comum no aparecimento do anestro pós-parto (Oliveira Filho, 2002).

A partição dos nutrientes utilizados pela vaca é priorizada seqüencialmente para metabolismo basal, atividade física, crescimento, reservas corporais básicas, lactação, acúmulo de reservas corporais, ciclo estral e início da gestação (Short et al., 1990), ressaltando-se que essa prioridade pode mudar de acordo com os estádios produtivos e reprodutivos do animal e da qualidade e quantidade de alimento oferecido. Alimentação de boa qualidade, no final do período de gestação e início da lactação, contribui para boa eficiência reprodutiva e produtiva do rebanho.

$\mathrm{O}$ escore de condição corporal tem sido utilizado para avaliar a condição nutricional dos bovinos e, conseqüentemente, estimar sua performance reprodutiva no pós-parto (Randel, 1990; DeRouen et al., 1994; Ferreira et al., 1997).
Vacas com bom escore da condição corporal (ECC) ao parto, igual ou superior a 5 a 6 pontos (escala de 1 a 9), apresentam melhor eficiência reprodutiva, caracterizada pelo menor intervalo de partos (Laflamme \& Connor, 1992; Osoro \& Wright, 1992; Rae et al. 1993; Schwalbach et al. 1997; Oltjen \& Sainz 1997; Valle et al., 1998), o que também pode ser observado em novilhas (DeRouen et al., 1994; Vizcarra et al., 1998).

A suplementação pré-parto proporciona melhor ECC ao parto e maior eficiência reprodutiva em vacas taurinas (Corah et al., 1975; Bellows \& Short, 1978; Marston et al., 1995; Sptizer et al., 1995), assim como em vacas mestiças (Fordyce et al., 1997; Oliveira Filho et al., 1997). No entanto, McSweeney et al. (1993) não observaram melhoria da performance reprodutiva em vacas mestiças suplementadas no pré e pós-parto. Vacas taurinas suplementadas no pósparto também melhoraram sua performance reprodutiva (Henricks et al., 1986; Marston et al., 1995; Sptizer et al., 1995). No entanto, em vacas zebuínas suplementadas no pós-parto, não se verificou melhoria da performance reprodutiva (Ruas et al., 2000; Nicodemo et al., 2001).

Vacas taurinas e mestiças mantidas em pastagens melhoradas no pré e pós-parto, independentemente de suplementação com concentrado ou não, tiveram índices de prenhez superiores em relação aos animais submetidos à pastagens naturais (Lobato et al., 1998ab).

Os níveis plasmáticos de metabólitos sanguíneos têm sido usados com o intuito de demonstrar seu efeitos sobre os bovinos, podendo a suplementação alterar o padrão destes metabólitos e de hormônios (Russel \& Wright, 1983; Ruas et al., 1998abc; Oliveira Filho et al., 2000).

O colesterol pode influenciar na performance reprodutiva dos bovinos, por ser precursor de hormônios esteróides importantes como a progesterona (Kappel et al., 1984; Ruegg et al., 1992b; Ruas et al., 1998b). Baixos níveis sangüíneos de colesterol diminuem sua concentração no ovário, podendo prejudicar a produção de hormônios esteróides (Cook et al., 1996). A maioria do colesterol sanguíneo é transportada por lipoproteínas de alta (HDL) e baixa densidade (LDL), que são captadas pelo tecido ovariano para a produção destes hormônios (Grummer \& Carroll, 1988).

O aumento de colesterol de vacas zebuínas no pós-parto pode estar associado à mobilização de 
reservas corporais para atender a demanda de nutrientes para lactação e mantença, principalmente em vacas que diminuem o ECC no pós-parto (Ruas et al, 1998c; Ruas et al., 2000ab). Também nas vacas holandesas em lactação, o colesterol aumentou no pós-parto, à medida que houve perda de ECC (Ruegg et al., 1992a).

Oliveira Filho (1999) observou, em vacas da raça Canchim, que as concentrações médias de colesterol nos animais suplementados no pré e pós-parto (186,31 e $179,73 \mathrm{mg} / \mathrm{dL}$, respectivamente) foram maiores que no grupo controle $(160,17 \mathrm{mg} / \mathrm{dL})$.

Vacas Nelore paridas sem suplementação e recebendo 1,0 ou $2,0 \mathrm{~kg}$ de concentrado (40,8\% PB), a pasto, durante 105 dias pós-parto, apresentaram concentrações médias de colesterol de 180,48; 181,81 e $184,74 \mathrm{mg} / \mathrm{dL}$ respectivamente (Ruas et al., 2000b).

O objetivo deste estudo foi de avaliar no pós-parto as variações do peso corporal, escore de condição corporal e dos níveis plasmáticos de colesterol total, além do desempenho reprodutivo de vacas da raça Guzerá suplementadas nos períodos pré e pós-parto e a viabilidade econômica dos tratamentos.

\section{Material e Métodos}

O trabalho foi desenvolvido na Fazenda de Ensino e Pesquisa da Faculdade de Engenharia de Ilha Solteira - UNESP localizada no município de Selvíria - MS, no período de 20 de agosto de 2001 a 22 de abril de 2002. A pluviosidade neste período foi de $1115,4 \mathrm{~mm}$, com temperatura média de $25,5^{\circ} \mathrm{C}$ e umidade relativa média do ar de $62,4 \%$.

Foram utilizadas 30 vacas da raça Guzerá no terço final de gestação, distribuídas de acordo com o número de partos, o peso corporal e a idade. A área experimental foi constituída de nove piquetes de Brachiaria decumbens com 2,8 hectares cada, providos de bebedouros e cocho coberto para sal mineral. Os animais foram manejados de forma rotacionada, permanecendo 14 dias em cada piquete, de modo a permitir que cada tratamento tivesse disponível um piquete para ocupação e dois em descanso.

Os animais foram divididos em três tratamentos com 10 repetições: PRE (suplementação pré parto); POS (suplementação pós-parto); SS (sem suplementação). A suplementação foi constituída de $1 \mathrm{~kg} / \mathrm{animal} /$ dia de concentrado com $16 \%$ de proteína bruta e $3000 \mathrm{kcal}$ de energia digestível $/ \mathrm{kg}$, fornecido em dois períodos $(0,5 \mathrm{~kg}$ de manhã e $0,5 \mathrm{~kg}$ à tarde) .
O sal mineral foi fornecido à vontade em cochos próprios. Os cochos para o fornecimento dos suplementos foram dimensionados em $70 \mathrm{~cm}$ linear por animal, para que todos os animais tivessem acesso ao concentrado.

Os animais foram desverminados e vacinados contra febre aftosa e clostridioses. Os pesos médios dos animais no início do período experimental (20 de agosto de 2001) foram de 453,9; 454,6 e 443,5 kg para os tratamentos PRE, POS e SS, respectivamente.

As vacas foram submetidas à estação de monta com touro anteriormente ao experimento, o que dificultou a padronização das vacas no mesmo estádio de gestação. As vacas do tratamento PRE tiveram períodos de suplementação variável (30 a 150 dias), e à medida que pariam foram colocadas junto com aquelas do tratamento SS, de modo que após o parto não fossem mais suplementadas. Dessa maneira, foram disponibilizados dois piquetes para o lote (PRE + SS), para não aumentar a lotação do piquete do tratamento SS. As vacas do POS foram suplementadas do parto até 112 dias pós-parto.

A pesagem das vacas foi realizada no início do experimento, após 24 horas do nascimento do bezerro e a cada 28 dias até 112 dias pós-parto, sempre acompanhada de avaliação de escore de condição corporal (ECC em que 1: muito magra e 9: muito gorda), segundo Nicholson \& Butterworth (1986) citado por Valle et al. (1998). As áreas avaliadas por palpação e inspeção visual, para determinação da cobertura muscular e de gordura, foram as costelas, os processos transversos das vértebras lombares, as extremidades dos ílios e ísquios e a região da inserção da cauda.

Para avaliação da eficiência reprodutiva das vacas, foram utilizados dois touros da raça Guzerá, após prévio exame andrológico, os quais foram colocados nos piquetes com as vacas logo após os partos até 112 dias pós-parto, de modo que um touro permaneceu nos tratamentos PRE e SS e outro no tratamento POS. As vacas foram observadas duas vezes ao dia, uma hora pela manhã e à tarde, sendo anotados o número da vaca e a data de cobertura. As vacas no $112^{\mathrm{o}}$ dia pós-parto foram retiradas do piquete e submetidas, após 45 dias, ao diagnóstico de gestação por palpação retal.

Coletas de sangue foram realizadas 24 horas pósparto e semanalmente até 112 dias, de manhã antes da suplementação, por punção da veia jugular, com agulhas de dimensão 30 x 20 e colhido em frascos de 
vidro sem anticoagulante. As amostras foram mantidas sob refrigeração durante 24 horas após a coleta para separação do soro sanguíneo e enviadas para análise laboratorial do colesterol total, que foi analisado em Laboratório Clínico, por meio de automatização no equipamento VP-SUPER SISTEM.

A análise econômica da suplementação foi realizada segundo metodologia adotada por Oliveira Filho (1999).

A análise estatística foi realizada com o auxílio do SAS (Statistical Analysis System), com o procedimento GLM (General Linear Model) (SAS, 1987).

O modelo adotado incluiu os efeitos de tratamento, animal e dias pós-parto. O delineamento utilizado foi semelhante a um esquema com parcelas subdivididas, tendo como fatores principais os tratamentos, animal dentro de tratamento e como fatores secundários os dias pós parto e a interação tratamento $\mathrm{x}$ dias pós-parto. Quando a interação foi significativa, o prosseguimento da análise se deu através do teste de Tukey. Para comparar fertilidade empregou-se o teste Qui-quadrado.

\section{Resultados e Discussão}

Os pesos corporal das vacas 24 horas após o parto foram de 477,80; 453,37 e 447,57 kg, respectivamente, para os grupos suplementados pré-parto (PRE), pós-parto (POS) e sem suplementação (SS) (Tabela 1). A suplementação pré-parto (30 a 150 dias) proporcionou aumento de peso das vacas ao parto $(\mathrm{P}<0,01)$, enquanto os animais dos tratamentos POS e SS mantiveram seu peso corporal. Os pesos das vacas durante o período experimental apresentaram interação dos dias pós-parto $\mathrm{x}$ tratamento $(\mathrm{P}<0,01)$ (Figura 1).

Nos primeiros 28 dias pós-parto, ocorreram perdas de peso para todos os grupos, provavelmente pelo aumento da exigência da lactação, acentuando o balanço energético negativo das vacas. A partir dos 28 dias, observou-se ganho de peso para o grupo POS e perda para os grupos SS e PRE, sendo que no grupo PRE esta perda foi mais acentuada. Mesmo com esta perda de peso, aos 28 dias pós-parto as vacas do tratamento PRE mostraram maior peso $(14,30 \mathrm{~kg})$ $(\mathrm{P}<0,01)$ em relação aos tratamentos POS $(13,37 \mathrm{~kg})$ e SS $(12,29 \mathrm{~kg})$, que não diferiram entre si. Aos 56 dias pós-parto, as vacas do tratamento POS apresentaram aumento de peso $(446,25 \mathrm{~kg})$ em relação aos 28 dias $(440,00 \mathrm{~kg})$, em razão da suplementação, não diferindo do grupo PRE $(462,80 \mathrm{~kg})$, apesar deste ter diminuído o peso, enquanto no grupo SS o peso continuou estável $(438,42 \mathrm{~kg})$, mas inferior $(\mathrm{P}<0,01)$ ao PRE.

As vacas dos tratamentos PRE e POS apresentaram pesos similares $(460,00$ e $457,50 \mathrm{~kg}$, respectivamente) aos 84 dias pós-parto porém superiores $(\mathrm{P}<0,01)$ ao $\mathrm{SS}(434,42 \mathrm{~kg})$. Aos 112 dias pós-parto, o peso vivo das vacas do PRE $(457,79 \mathrm{~kg})$ não apresentou diferença $(\mathrm{P}<0,01)$ em relação ao POS e o SS (461,87 e 436,57 kg, respectivamente), mas o peso das vacas do POS foi maior $(\mathrm{P}<0,01)$ que do $S S$. Os animais dos tratamentos PRE e SS perderam peso $(-20,01 \mathrm{e}-11,00 \mathrm{~kg}$, respectivamente), do parto até 112 dias pós-parto, enquanto para o tratamento POS ganhou peso $(8,5 \mathrm{~kg})$. Apesar do comportamento diferente dos tratamentos até 112 dias pós-parto, os mesmos apresentaram os pesos médios de 457,79; 461,87 e $436,37 \mathrm{~kg}$, respectivamente, para os tratamentos PRE, POS e SS (Tabela 1).

Resultados semelhantes foram encontrados por Oliveira Filho (1999) trabalhando com vacas Canchim sem suplementação, suplementadas nos períodos pré e pós-parto, ou apenas no pós-parto. Os autores observaram perda de peso até os 30 dias, sendo que as vacas sem suplementação mantiveram a perda de peso até 60 dias, enquanto as do tratamento pós-parto ganharam peso após os 30 dias. Efeito benéfico da

Tabela 1 - Médias da variação do peso corporal de vacas da raça Guzerá no período pós-parto submetidas à suplementação antes (PRE) e após o parto (POS) e sem suplementação (SS)

Table 1 - Averages of body weight variation of cows of Guzera breed in the postpartum period submitted to supplementation before (PRE) and after the parturition (POS) and without supplementation (SS)

\begin{tabular}{cccc}
\hline Dias pós-parto & \multicolumn{3}{c}{$\begin{array}{c}\text { Tratamentos } \\
\text { Treatments }\end{array}$} \\
\cline { 2 - 4 } & \multicolumn{3}{c}{ PRE postpartum } \\
\cline { 2 - 4 } & & $\mathrm{kg}$ & $\mathrm{SS}$ \\
\cline { 2 - 4 } Parto & $477,80^{\mathrm{a}}$ & $453,37^{\mathrm{b}}$ & $447,57^{\mathrm{b}}$ \\
Parturition & & & \\
28 & $463,50^{\mathrm{a}}$ & $440,00^{\mathrm{b}}$ & $435,28^{\mathrm{b}}$ \\
56 & $462,80^{\mathrm{a}}$ & $446,25^{\mathrm{ab}}$ & $438,42^{\mathrm{b}}$ \\
84 & $460,00^{\mathrm{a}}$ & $457,50^{\mathrm{a}}$ & $434,42^{\mathrm{b}}$ \\
112 & $457,79^{\mathrm{ab}}$ & $461,87^{\mathrm{a}}$ & $436,57^{\mathrm{b}}$ \\
\hline
\end{tabular}

Letras diferentes na mesma linha diferem pelo teste Tukey a $1 \%$.

Different letters in the same line differ by Tukey test at the level of $1 \%$. 
suplementação pós-parto também foi verificado por McSweeney et al. (1993) e por Ruas et al. (2000a), os quais verificaram aumento de peso das vacas suplementadas após o parto.

Fordyce et al. (1997) observaram que a suplementação pré-parto proporcionou menor perda de peso e de ECC no pós-parto. Estes dados diferem dos encontrados para as vacas Guzerá do tratamento PRE, que perderam mais peso e ECC no pós-parto em relação ao tratamento SS e POS.

$\mathrm{O}$ ECC apresentou interação dias pós-parto $\mathrm{x}$ tratamento $(\mathrm{P}<0,01)$. Ao parto, o ECC das vacas do tratamento PRE $(6,20)$ foi maior $(\mathrm{P}<0,01)$ que dos tratamentos POS $(5,50)$ e SS $(5,28)$, que não diferiram entre si (Tabela 2).

Nos primeiros 28 dias, ocorreu perda de ECC para os grupos PRE e SS $(0,20$ e 0,14 unidades, respectivamente), fato que não ocorreu para o grupo POS, que ganhou ECC (0,12 unidades). A partir dos 28 dias, observou-se mantença ou ganho discreto para o grupo POS e perda para os grupos SS e PRE, sendo que no grupo PRE esta perda foi mais acentuada (Figura 2). Os animais dos tratamentos PRE e SS (sem suplementação no pós-parto) mobilizaram suas reservas corporais para atender suas exigências nutricionais, o que resultou na diminuição do ECC pós-parto.

O ECC das vacas do tratamento POS, aos 28 e 56 dias pós-parto (5,62 e 5,62, respectivamente), não

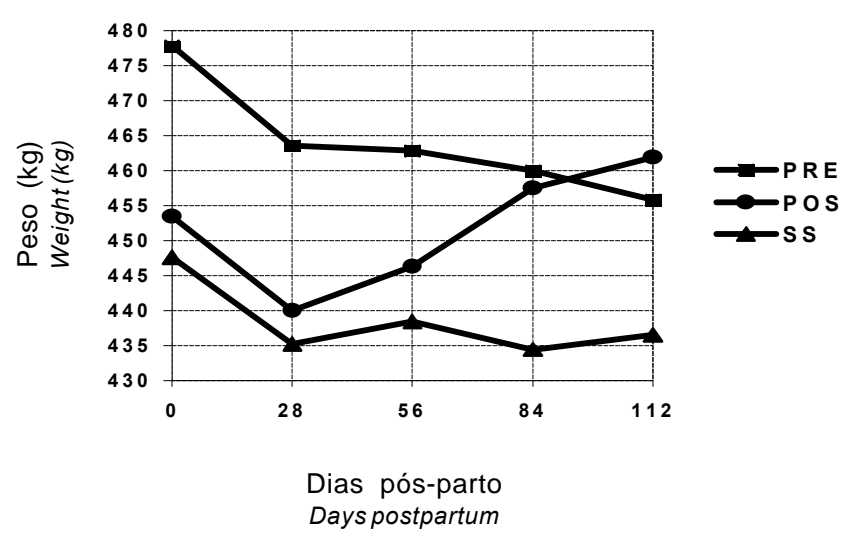

Figura 1 - Peso corporal de vacas da raça Guzerá no período pós-parto submetidas à suplementação antes (PRE) e após o parto (POS) e sem suplementação (SS).

Figure 1 - Body weight of cows of Guzerá breed in the postpartum period submitted to supplementation before (PRE) and after the parturition (POS) and without supplementation (SS). diferiu $(\mathrm{P}>0,01)$ dos tratamentos PRE e SS. No entanto, o ECC das vacas do tratamento PRE manteve-se significativamente superior em relação ao das vacas do tratamento SS, dos 28 até 84 dias pósparto (6,00 a 5,38 e 5,14 a 4,78 , respectivamente). Aos 84 dias pós-parto o ECC dos tratamentos PRE e $\operatorname{POS}(5,44$ e 5,56, respectivamente) não diferiram entre si $(\mathrm{P}>0,01)$, mas foram superiores $(\mathrm{P}<0,01) \mathrm{em}$ relação ao tratamento $\mathrm{SS}(4,78)$.

O ECC dos animais do tratamento POS se manteve praticamente constante do parto até 84 dias, aumentando até os 112 dias pós-parto. No entanto, aos 112 dias pós-parto, o ECC das vacas do tratamento PRE $(5,38)$ não diferiu $(\mathrm{P}>0,01)$ do POS e SS (5,66 e 4,78, respectivamente), ao contrário das vacas do tratamento POS que apresentaram ECC superior $(\mathrm{P}<0,01)$ ao tratamento SS.

O ECC das vacas do tratamento PRE manteve-se superior $(\mathrm{P}<0,01)$ ao SS até 84 dias pós-parto, e somente aos 112 dias pós-parto não houve diferença $(\mathrm{P}>0,01)$ (5,38 e 4,78, respectivamente). No entanto, o ECC das vacas do tratamento POS foi semelhante ao PRE, dos 28 até 112 dias pós-parto. As vacas do tratamento POS apresentaram ECC maior $(\mathrm{P}<0,01)$ dos 84 até 112 dias pós-parto em relação ao tratamento SS.

Ruas et al. (2000) também observaram que vacas com ECC ao parto semelhantes $(3,93$ e 3,87), ao receberem suplementação no pós-parto aumentaram

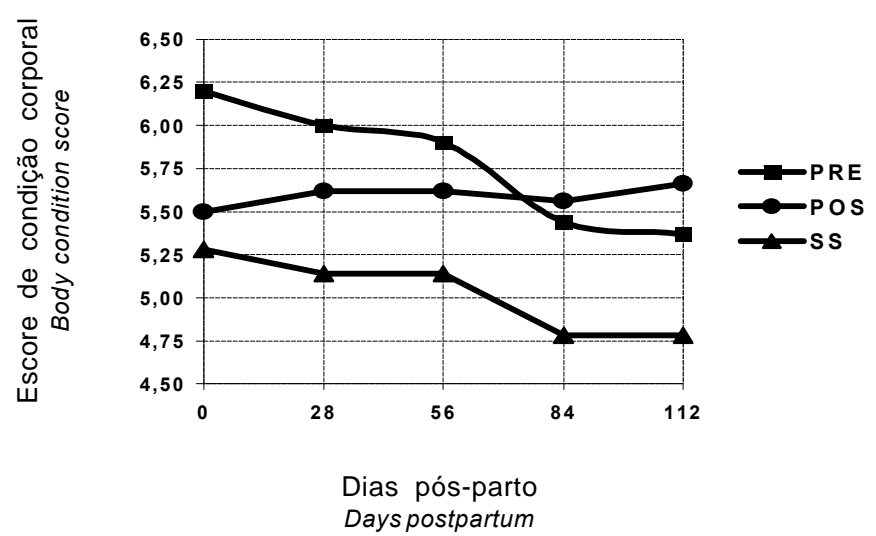

Figura 2 - Escore de condição corporal de vacas da raça Guzerá no período pós-parto submetidas à suplementação antes (PRE) e após o parto (POS) e sem suplementação (SS).

Figure 2 - Body condition score of cows of Guzerá breed in the postpartum period submitted to supplementation before (PRE) and after the parturition (POS) and without supplementation (SS). 
de forma progressiva o ECC até 120 dias pós-parto.

Laflamme \& Connor (1992) não obtiveram interação entre o ECC e a suplementação no pós-parto, mas o ECC e o peso foram significativamente maiores do parto até 210 dias para vacas com ECC moderado, quando comparado às vacas com ECC baixo.

Os níveis plasmáticos de colesterol foram influenciados $(\mathrm{P}<0,01)$ pelos dias pós-parto, mas não pelos tratamentos (Tabela 3; Figura 3). Verificaram-se aumentos dos níveis de colesterol em todos os tratamentos, à medida que transcorreram os dias pósparto, com valores oscilando de 108,05 a 118,27 mg/dL ao parto e 174,22 a 182,71 mg/d aos 112 dias pósparto (Tabela 3).

Resultados semelhantes foram observados por Ruas et al. (2000a) que, mensurando o colesterol sanguíneo de vacas zebuínas, observaram que vacas Guzerá aumentaram os níveis de colesterol plasmático aolongo do período pós-parto, de 148,64 a 194,47 mg/dL. Ruas et al. (2000b) também observaram que o colesterol plasmático aumentou com o transcorrer dos dias pós-parto, com médias de 180,48 a 184,74, em animais ingerindo diferentes quantidades de suplementos protéicos no pós-parto, ( 0 a $2 \mathrm{~kg} / \mathrm{dia})$. Ruas et al. (2000b) relacionaram o aumento dos níveis de colesterol com a produção de leite e partição de nutrientes durante a lactação, em que ácidos graxos poderiam se incorporar à gordura do leite ou serem utilizados para atender à demanda energética, à me-

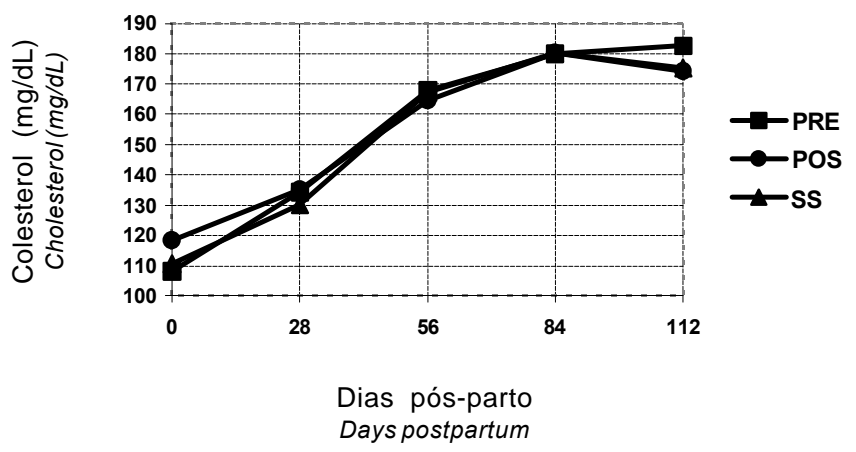

Figura 3 - Níveis de colesterol de vacas da raça Guzerá no período pós-parto submetidas à suplementação antes (PRE) e após o parto (POS) e sem suplementação (SS).

Figure 3 - Levels of cholesterol of cows of Guzera breed in the postpartum period submitted to supplementation before (PRE) and after the parturition (POS) and without supplementation (SS). dida que aumentou o número de dias pós-parto.

Concordando com os dados encontrados no presente estudo, Ruegg et al. (1992a) e Oliveira Filho (1999) também observaram aumentos progressivos de colesterol plasmático no pós-parto de vacas em lactação.

Ao final do período de 112 dias pós-parto, os índices de prenhez não diferiram $(\mathrm{P}>0,05)$ entre os tratamentos PRE, POS e SS, com médias de 50,00; 62,20 e $57,14 \%$, respectivamente. A média de prenhez dos tratamentos foi de $56,44 \%$. Os baixos índices de prenhez podem ser devido ao efeito deletério da amamentação, considerando-se que a maioria dos animais de todos os tratamentos apresentaram boa condição física e adequado ECC.

Freitas (2000) observou efeito deletério da amamentação sobre a taxa de prenhez de vacas Guzerá, quando as vacas com a presença do bezerro apresentaram taxas de $60 \%$ de prenhez, contra $80 \%$ daquelas que permaneceram apenas uma hora por dia com o bezerro.

A falta de resposta da suplementação sobre o índice de prenhez no tratamento POS concorda com os achados de Ruas et al. (2000b) e Nicodemo et al. (2001), que também não encontraram melhoria significativa na eficiência reprodutiva de vacas Nelore suplementadas no pós-parto.

$\mathrm{O}$ ECC ao parto não influenciou nos índices de prenhez das vacas submetidas aos diferentes tratamentos, possivelmente pelo fato de não ter ocorrido grandes variações dos ECC entre as vacas dos diferentes tratamentos (Tabela 4) e talvez por serem vacas zebuínas. Isto pôde ser observado por Schwalbach et al. (1997), que não verificaram melhoria da fertilidade em vacas com ECC abaixo de 6,25 pontos. No entanto, Osoro \& Wright (1992) observaram que o melhor ECC ao parto foi o principal fator na performance reprodutiva, com maiores índices de prenhez. Rae et al. (1993), DeRouen et al. (1994) e Spitzer et al. (1995) também apontaram o ECC ao parto como indicador do desempenho reprodutivo no pós-parto.

Neste estudo, foram calculados apenas os custos com suplementação, considerando que os demais custos para produção de bezerros foram iguais, segundo Oliveira Filho (1999). As vacas do tratamento PRE tiveram variação no período de suplementação (30 a 150 dias no pré-parto), no qual se adotou período médio de 90 dias de suplementação para efeito de calculo dos custos. As vacas do tratamento POS receberam suplementação por 112 dias pós-parto. As 
Tabela 2 - Médias da variação do escore de condição corporal (ECC- 1 a 9) de vacas da raça Guzerá no período pós-parto submetidas à suplementação antes (PRE) e após o parto (POS) e sem suplementação (SS)

Table 2 - Averages of the variation of the body condition score (BCS) of cows of Guzera breed in the postpartum period submitted to supplementation before (PRE) and after the parturition (POS) and without supplementation (SS)

\begin{tabular}{|c|c|c|c|}
\hline \multirow[t]{2}{*}{$\begin{array}{l}\text { Dias pós-parto } \\
\text { Days postpartum }\end{array}$} & \multicolumn{3}{|c|}{$\begin{array}{c}\text { Tratamentos } \\
\text { Treatments }\end{array}$} \\
\hline & PRE & POS & SS \\
\hline Parto & $6,20^{\mathrm{a}}$ & $\begin{array}{c}C C(1-\mathrm{C} \\
5,50^{\mathrm{b}}\end{array}$ & $5,28^{\mathrm{b}}$ \\
\hline \multicolumn{4}{|l|}{ Parturition } \\
\hline 28 & $6,00^{\mathrm{a}}$ & $5,62^{\mathrm{ab}}$ & $5,14^{\mathrm{b}}$ \\
\hline 56 & $5,90^{\mathrm{a}}$ & $5,62^{a b}$ & $5,14^{\mathrm{b}}$ \\
\hline 84 & $5,44^{\mathrm{a}}$ & $5,56^{\mathrm{a}}$ & $4,78^{\mathrm{b}}$ \\
\hline 112 & $5,38^{a b}$ & $5,66^{\mathrm{a}}$ & $4,78^{b}$ \\
\hline
\end{tabular}

Letras diferentes na mesma linha diferem pelo teste Tukey a $1 \%$.

Different letters in the same line differ by Tukey test at the level of $1 \%$.

Tabela 4 - Custos adicionais com a suplementação antes (PRE) e após o parto (POS) e sem suplementação (SS) de vacas da raça Guzerá

Table 4 - Additional costs with the supplementation before (PRE) and after parturition (POS) and without supplementation (SS) of cows of Guzerá breed

\begin{tabular}{|c|c|c|c|}
\hline \multirow{2}{*}{$\begin{array}{l}\text { Discriminação } \\
\text { Discrimination }\end{array}$} & \multicolumn{3}{|c|}{$\begin{array}{c}\text { Tratamentos } \\
\text { Treatments }\end{array}$} \\
\hline & PRE & POS & $\mathrm{SS}$ \\
\hline Número de vacas & 10 & 10 & 10 \\
\hline $\begin{array}{l}\text { Number of cows } \\
\text { Índice de prenhez }(\%) \\
\text { Rate pregnancy }(\%)\end{array}$ & 50,00 & 62,20 & 57,14 \\
\hline $\begin{array}{l}\text { Rate pregnancy (\%) } \\
\text { Número de bezerros } \\
\text { Number of calves }\end{array}$ & 5,00 & 6,20 & 5,71 \\
\hline $\begin{array}{l}\text { Preço bezerro }(\mathrm{R} \$)^{*} \\
\text { Price calf }(R \$)^{*}\end{array}$ & 350,00 & 350,00 & 350,00 \\
\hline $\begin{array}{l}\text { Receita }(\mathrm{R} \$) \\
\text { Proceeds }(R \$)\end{array}$ & 1750,00 & 2170,00 & 1998,50 \\
\hline $\begin{array}{l}\text { Suplemento }(\mathrm{kg} / \mathrm{dia}) \\
\text { Supplement }(\mathrm{kg} / \text { day })\end{array}$ & 1,00 & 1,00 & - \\
\hline $\begin{array}{l}\text { Período (dias) } \\
\text { Period (days) }\end{array}$ & 90 & 112 & - \\
\hline $\begin{array}{l}\text { Custo/kg** } \\
\text { Cost } / \mathrm{kg}^{* *}\end{array}$ & 0,16 & 0,16 & - \\
\hline $\begin{array}{l}\text { Custo adicional }(\mathrm{R} \$) \\
\text { Additional costs }(R \$)\end{array}$ & 144,00 & 179,20 & - \\
\hline $\begin{array}{l}\text { Saldo financeiro }(\mathrm{R} \$) \\
\text { Financial balance }(R \$)\end{array}$ & 1606,00 & 1990,80 & 1998,50 \\
\hline $\begin{array}{l}\text { Análise comparativa do } \\
\text { PRE e POS com o SS (R\$) } \\
\text { Comparative analysis } \\
\text { between PRE and POS } \\
\text { and } S S(R \$)\end{array}$ & $-392,50$ & $-7,70$ & - \\
\hline
\end{tabular}

*BM\&F e CEPEA-ESALQ.
Tabela 3 - Médias da variação do colesterol plasmático $(\mathrm{mg} / \mathrm{dL})$ de vacas da raça Guzerá no período pós-parto submetidas à suplementação antes (PRE) e após o parto (POS) e sem suplementação (SS)

Table 3 - Averages of the variation of the serious cholesterol $(\mathrm{mg} / \mathrm{dL})$ of cows of Guzera breed in the postpartum period submitted to supplementation before (PRE) and after the parturition (POS) and without supplementation (SS)

\begin{tabular}{cccc}
\hline Dias pós-parto & \multicolumn{3}{c}{$\begin{array}{c}\text { Tratamentos } \\
\text { Treatments }\end{array}$} \\
\cline { 2 - 4 } & \multicolumn{3}{c}{ POS postpartum } \\
\cline { 2 - 4 } & PRE & SS \\
\hline & & 118,27 & 110,64 \\
Parto & 108,05 & & \\
Parturiton & & 135,26 & 130,15 \\
28 & 134,33 & 164,49 & 167,54 \\
56 & 167,79 & 180,28 & 180,39 \\
84 & 180,14 & 174,22 & 175,21 \\
112 & 182,71 & & \\
\hline
\end{tabular}

vacas dos tratamentos PRE e POS tiveram ingestão de $1,00 \mathrm{~kg} /$ dia de suplemento, com um custo médio de $\mathrm{R} \$ 0,16 / \mathrm{kg}$. O preço do bezerro desmamado (8-10 meses de idade) foi avaliado em torno de $\mathrm{R} \$ 350,00$ (Tabela 4). Analisando comparativamente os saldos financeiros dos tratamentos PRE e POS com o tratamento SS, pode-se obter os seguintes resultados:

PRE - SS $=1606,00-1998,50=-\mathbf{R} \$ \mathbf{3 9 2 , 5 0}$

POS - SS $=1990,80-1998,50=-\mathbf{R} \$ \mathbf{7 , 7 0}$

Nas condições em que foi desenvolvido este experimento, a suplementação antes ou após o parto de vacas da raça Guzerá mostrou saldos financeiros negativos quando comparado às vacas sem suplementação. No entanto, o tratamento POS teve tendência em aumentar os índices de prenhez, em relação aos demais tratamentos, a menor custo, quando comparado ao tratamento PRE.

\section{Conclusões}

A suplementação no pré-parto melhorou o peso corporal e escore de condição corporal ao parto.

A suplementação no pós-parto proporcionou aumento do peso corporal e do escore de condição corporal do parto até 112 dias.

Os níveis de colesterol total aumentaram com o transcorrer dos dias pós-parto, independentemente da suplementação pré e pós-parto. 
Os índices de prenhez não foram influenciados pela suplementação pré e pós-parto.

A suplementação pré e pós-parto não foi viável economicamente.

\section{Literatura Citada}

BELLOWS, R.A.; SHORT, R.E. Effects of precalving feed level on birth weight, calving, difficulty and subsequent fertility. Journal of Animal Science, v.46, p.1522-1528, 1978.

COOK, C.M.; FOGWELL, R.L.; MENON, K.M. et al. Concentrations of cholesterol in plasma affect concentrations of cholesterol and progesterone in bovine corpus luteum. Journal of Animal Science, v.74, suppl. 1, p.222, 1996 (Abstracts, 459).

CORAH, L.R.; DUNN, T.G.; KALTENBACH, C.C. Influence of prepartum nutrition on the reproductive performance of beef females and the performance of their progeny. Journal of Animal Science, v.41, p.819-824, 1975.

DeROUEN, S.M.; FRANKE, D.E.; MORRISON, D.G. et al. Prepartum body condition and weight influences on reproductive performance of first - calf beef cows. Journal of Animal Science, v.72, p.1119-1125, 1994.

FERREIRA, M.B.D.; LOPES, P.C.; ANDRADE, V.J. et al. Escore corporal e anestro pós parto em primíparas zebu. Revista Brasileira de Reprodução Animal, v.21, n.2, p.114-116, 1997.

FORDYCE, G.; FITZPATRICK, L.A.; MULLIS, T.J. et al. Prepartum supplementation effects on growth and fertility in Bos indicus- cross cows. Australian Journal of Experimental Agriculture, v.37, p.141-149, 1997.

FREITAS, M.S. Efeitos da interrupção temporária do aleitamento, sobre o desempenho reprodutivo de vacas Guzerá. Ilha Solteira: Universidade Estadual Paulista, 2000. 33p. Dissertação (Mestrado em Zootecnia) - Faculdade de Engenharia de Ilha Solteira/Universidade Estadual Paulista, 2000.

GRUMMER, R.R.; CARROLL, D.J. A review of lipoprotein cholesterol metabolism: importance to ovarian function. Journal of Animal Science, v.66, p.3160-3170, 1988.

HENRICKS, D.M.; RONE, J.D.; FERRELL, C.L. et al. A note on the effect of nutrition on ovulation and ovarian follicular populations in the individually fed post-partum beef heifer. Animal Production, v.46, p.557-560, 1986.

KAPPEL, L.C.; INGRAHAM, R.H.; MORGAN, E.B. et al. Relationship between fertility and blood glucose and cholesterol concentrations in Hostein cows. Animal Journal Veterinary Research, v.45, n.12, p.2607-2612, 1984.

LAFLAMME, L.F.; CONNOR, M.L. Effect of postpartum nutrition and cow body condition at parturition on subsequent performance of beef cattle. Canadian Journal of Animal Science, v.72, n.4, p.843-851, 1992.

LOBATO, J.F.P.; DERESZ, F.; LEBOUTE, E.M. et al. Pastagens melhoradas e suplementação alimentar no comportamento reprodutivo de vacas de corte primíparas. Revista Brasileira de Zootecnia, v.27, n.1, p.47-53, 1998 a.

LOBATO, J.F.P.; ZANOTTA JUNIOR, R.L.D.; PEREIRA NETO, O.A. Efeitos das dietas pré e pós-parto na eficiência reprodutiva de vacas primíparas de corte. Revista Brasileira de Zootecnia, v.27, n.5, p.857-862, 1998 b.

MARSTON, T.T.; LUSBY, K.S.; WETTEMAN, R.P. et al.
Effects of feeding energy or protein supplements before or after calving on performance of spring-calving cows grazing native range. Journal of Animal Science, v.73, p.657-664, 1995.

McSWEENEY, C.S.; FITZPATRICK, L.A.; D'OCCHIO, M.J. et al. Reducing post-partum anoestrous interval in first - calf Bos indicus crossbred beef heifers. I. Effects of pre- and postpartum suplementation strategies. Australian Journal Agricultural Research, v.44, p.1063-1077, 1993.

NICODEMO, M.L.F.; MORAES, S.S., THIAGO, L.R.L. et al. Desempenho de vacas Nelore em pastos de braquiária suplementados com fósforo/cálcio e/ou proteína/energia durante a seca. In: REUNIÃO ANUAL DA SOCIEDADE BRASILEIRA DE ZOOTECNIA, 38., 2001, Piracicaba. Anais... Piracicaba: Fundação de Estudos Agrários Luiz de Queiroz, 2001.p.1095-1096.

OLIVEIRA FILHO, B.D.; GAMBARINI, M.L.; TONIOLLO, G.H. Efeitos da suplementação nutricional pré e pós parto e da condição corporal ao parto sobre a reprodução, em vacas de corte. Revista Brasileira de Reprodução Animal, v.21, n.2, p.112-113, 1997.

OLIVEIRA FILHO, B.D. Efeitos da suplementação pré e pós parto sobre parâmetros reprodutivos e metabólicos em vacas da raça Canchim. Jaboticabal: Universidade Estadual Paulista, 1999. 110p. Tese (Doutorado em Zootecnia) Universidade Estadual Paulista, 1999.

OLIVEIRA FILHO, B.D.; TONIOLLO, G.H.; GAMBARINI, M.L. et al. Efeitos da suplementação nutricional pré e pós parto sobre níveis séricos de colesterol, triglicerídeos e lipídeos totais em vacas da raça Canchim. In: REUNIÃO ANUAL DA SOCIEDADE BRASILEIRA DE ZOOTECNIA, 37., 2000, Viçosa, MG. Anais... Viçosa, MG: Sociedade Brasileira de Zootecnia/ [2000]. CD - ROM.

OLIVEIRA FILHO, B.D. Interrelações nutrição e reprodução em bovinos de corte. In: SIMPÓSIO GOIANIO SOBRE MANEJO E NUTRIÇÃO DE BOVINOS DE CORTE, 4., 2002, Goiânia. Anais... Goiânia: Colégio Brasileiro de Nutrição Animal, 2002. p.175-184.

OLTJEN, J.W.; SAINZ, R.D. Strategic supplementation of range beef cows: split feeding by body and stocking rate. Journal of Animal Science, v.75, suppl. 1, p.250, 1997, (Abstracts, 452).

OSORO, K.; WRIGHT, I.A. Effect of body condition, live weight, breed, age, calf performance, and calving date on reproductive performance of spring-calving beef cows. Journal of Animal Science, v.70, p.1661-1666, 1992.

PIRES, A.V.; SUSIN, I. Relações entre nutrição e reprodução. In: SIMPÓSIO SOBRE PRODUÇÃO ANIMAL, 9., 1996, Piracicaba. Anais... Piracicaba: Fundação de Estudos Agrários Luiz de Queiroz, 1997. p.69-96.

RAE, D.O.; KUNKLE, W.E.; CHENOWETH, P.J. et al. Relationship of parity and body condition score to pregnancy rates in Florida beef cattle. Theriogenology, v.39, p.1143-1152, 1993.

RANDEL, R.D. Nutrition and postpartum rebreeding in cattle. Journal of Animal Science, v.68, p.853-862, 1990

RUAS, J.R.M.; TORRES, C.A.A.; CARVALHO, G.R. et al. Níveis plasmáticos de colesterol, glicose e uréia em vacas de raças zebuínas. In: REUNIÃO ANUAL DA SOCIEDADE BRASILEIRA DE ZOOTECNIA, 35., 1998, Botucatu. Anais... Botucatu: Sociedade Brasileira de Zootecnia, 1998a. p.175-177.

RUAS, J.R.M.; TORRES, C.A.A.; SANTOS, M.D. et al. Variações nos níveis plasmáticos de colesterol, glicose e uréia 
em vacas de raças zebuínas com diferente escore corporal. In: REUNIÃO ANUAL DA SOCIEDADE BRASILEIRA DE ZOOTECNIA, 35., 1998, Botucatu. Anais... Botucatu: Sociedade Brasileira de Zootecnia, 1998b. p.178-180.

RUAS, J.R.M.; TORRES, C.A.A.; MACHADO, G.V. et al. Influência do "status" reprodutivo nos níveis plasmáticos de colesterol, glicose e uréia, e no escore corporal em vacas de raças zebuínas. In: REUNIÃO ANUAL DA SOCIEDADE BRASILEIRA DE ZOOTECNIA, 35., 1998, Botucatu. Anais... Botucatu: Sociedade Brasileira de Zootecnia, 1998c. p.181-83.

RUAS, J.R.M.; TORRES, C.A.A.; VALADARES FILHO, S.B. et al. Efeito da suplementação proteíca a pasto sobre consumo de forragens, ganho de peso e condição corporal, em vacas Nelore. Revista Brasileira de Zootecnia, v.29, n.3, p.930934, 2000.

RUAS, J.R.M.; TORRES, C.A.A.; BORGES, L.E. et al. Concentrações plasmáticos de colesterol, glicose e uréia em vacas zebuínas, em relação à condição corporal e ao "status" reprodutivo. Revista Brasileira de Zootecnia, v.29, n.6, supl. 1, p.2036-2042, 2000a.

RUAS, J.R.M.; TORRES, C.A.A.; BORGES, L.E. et al. Efeito da suplementação proteíca a pasto sobre eficiência reprodutiva e concentrações sanguíneas de colesterol, glicose e uréia, em vacas Nelore. Revista Brasileira de Zootecnia, v.29, n.6, supl. 1, p.2043-2050, 2000b.

RUEGG, P.L.; GOODGER, W.J.; HOLMBERG, C.A. et al. Relation among body condition score, milk production and serum urea nitrogen and cholesterol concentrations in highproducing Holstein dairy cows in early lactation. Animal Journal Veterinary Research, v.53, n.1, p.5-9, 1992a.

RUEGG, P.L.; GOODGER, W.J.; HOLMBERG, C.A. et al. Relation among body condition score, serum urea nitrogen and cholesterol concentrations, and reproductive performance in high-producing Holstein dairy cows in early lactation. Animal Journal Veterinary Research, v.53, n.1, p.10-14, 1992b.

RUSSEL, A.J.F.; WRIGHT, I.A. The use of blood metabolites in the determination of energy status in beef cows. Animal Production, v.37, p.335-343, 1983.
SANTOS, J.E.P.; AMSTALDEN, M. Effects of nutrition on bovine reproduction. Arquivos da Faculdade Veterinária da UFRGS, v.26, n.1, p.19-89, 1998.

STATISTIC ANALYSES SYSTEM - SAS. Guide for personal computers. 6.ed. Cary: 1987. 1028p.

SCHWALBACH, L.M.; PEREIRA, C.M.L.; ESCRIVAO, R. et al. Determinação da condição corporal e sua relação com a fertilidade em cinco fazendas de gado bovino de corte em Moçambique. Veterinária-Técnica, v.7, n.5, p.18-20, 1997.

SHORT, R.E.; BELLOWS, R.A.; STAIGMILLER, R.B. et al.Physiological mechanisms controlling anestrus and infertility in postpartum beef cattle, Journal of Animal Science, v.68, p.799-816, 1990.

SPTIZER, J.C.; MORRISON, D.G.; WETTEMAN, R.P. et al. Reproductive responses and calf birth and weaning weights as affected by body condition at parturition and postpartum weight gain in primiparous beef cows. Journal of Animal Science, v.73, p.1251-1257, 1995.

STABENFELDT, G.H. Reprodução e lactação In: CUNNINGHAM, J. (Ed.) Tratado de fisiologia veterinária. Rio de Janeiro: Guanabara Koogan, 1993. p.317.

VALLE, E.R.; ANDREOTTI, R.; THIAGO, L.R.L.S. Estratégias para aumento da eficiência reprodutiva e produtiva em bovinos de corte. Campo Grande: EMBRAPACNPGC, 1998. p.19.

VIZCARRA, J.A.; WETTEMANN, R.P.; SPTIZER, J.C. et al. Body condition at parturition and postpartum weight gain influence luteal activity and concentrations of glucose, insulin, and nonesterified fatty acids in plasma of primiparous beef cows. Journal of Animal Science, v.76, p.927-936, 1998.

Recebido em: 18/10/02

Aceito em: 04/08/03 